\title{
FLUTUAÇÃO DIÁRIA E ESTACIONAL DE NITRATO E AMÔNIO EM UM ARGISSOLO VERMELHO DISTRÓFICO TÍPICO ${ }^{(1)}$
}

\author{
Naracelis Poletto ${ }^{(2)}$, Daniel Santos Grohs ${ }^{(2)}$ \& Claudio Mario Mundstock ${ }^{(3)}$
}

\section{RESUMO}

Os íons $\mathrm{NH}_{4}{ }^{+} \mathrm{e} \mathrm{NO}_{3}{ }^{-}$resultantes do processo de mineralização do $\mathrm{N}$ orgânico no solo são as formas predominantes de $\mathrm{N}$ mineral disponíveis às plantas. A proporção desses íons e sua distribuição no solo é variável, com flutuações diárias e oscilações sazonais ao longo do ano. Este trabalho avaliou a variação dos teores de $\mathrm{NH}_{4}{ }^{+} \mathrm{e} \mathrm{NO}{ }_{3}{ }^{-}$ em duas áreas adjacentes, sendo uma delas cultivada com grãos (cevada, soja e cevada) no sistema de plantio direto há mais de dez anos. Esta área recebeu, ao longo do tempo, fertilizantes à base de $\mathrm{P} \mathrm{e} \mathrm{K}$ e apresentava $\mathrm{pH}$ 6,0. A outra área foi mantida sem cultivo (campo limpo), com predominância de gramíneas, com baixa fertilidade do solo e pH 4,8. O estudo foi realizado em um Argissolo Vermelho distrófico típico na Estação Experimental Agronômica da UFRGS, em Eldorado do Sul-RS, nos anos agrícolas de 2002 e de 2003. As amostragens de solo, de caráter estacional na área cultivada (31/05/2002 a 23/10/2003) e não-cultivada (13/06/2002 a 17/10/2002), foram feitas, em média, a cada dez dias. Amostragens de caráter diário (16/05 a 30/09 de 2003) foram realizadas em intervalos de um a três dias. Em ambos os casos, as amostragens de solo foram feitas na camada superficial de 0-0,2 $\mathrm{m}$ de profundidade, em áreas que não receberam adubação nitrogenada durante todo o período. As avaliações estacionais mostraram que o $\mathrm{N}$ mineral $\left(\mathrm{NH}_{4}{ }^{+}+\mathrm{NO}_{3}{ }^{-}\right)$elevouse da primavera até o verão e decresceu no período outono/inverno tanto em 2002 quanto em 2003. As variações estacionais evidenciaram que a mineralização do $\mathbf{N}$ acompanhou, aproximadamente, as oscilações na temperatura média do período analisado. As avaliações diárias mostraram que os teores de $\mathrm{N}$ mineral variaram de um dia para outro, sendo as maiores variações ocasionadas pelas chuvas. $\mathrm{Na}$ área com vegetação nativa, o $\mathrm{N}$ teve comportamento semelhante ao observado nas áreas cultivadas com cevada e soja. A proporção 1:1 dos íons $\mathrm{NH}_{4}{ }^{+}$e $\mathrm{NO}_{3}^{-}$, observada tanto no solo cultivado quanto naquele mantido sob vegetação nativa, indica que há um equilíbrio entre as duas formas iônicas, independente do pH e do nível de fertilidade do solo.

Termos de indexação: nitrogênio, sistema plantio direto, temperatura, precipitação, pH.

\footnotetext{
(1) Recebido para publicação em agosto de 2007 e aprovado em abril de 2008.

(2) Engenheiro-Agrônomo, Estudante do Programa de Pós-Graduação em Fitotecnia, Faculdade de Agronomia, Universidade Federal do Rio Grande do Sul - UFRGS. Av. Bento Gonçalves 7712, Caixa Postal 15100, CEP 91501-970 Porto Alegre (RS). E-mails: narapoletto@yahoo.com.br; dan_gro@hotmail.com

(3) Engenheiro-Agrônomo, PhD, Professor do Programa de Pós Graduação em Fitotecnia, UFRGS. E-mail: cmmundst@ufrgs.br
} 


\title{
SUMMARY: AMMONIUM AND NITRATE SEASONAL AND DAILY FLUCTUATION IN A TYPICAL RED DYSTHROPIC ARGISOL
}

\begin{abstract}
Ammonium and nitrate ions from soil organic $N$ mineralization are the predominant $N$ mineral forms available to plants. The ion ratio and distribution along the soil profile show daily fluctuations and oscillate seasonally. $\mathrm{NH}_{4}{ }^{+}$and $\mathrm{NO}_{3}{ }^{-}$soil content were evaluated in two adjacent plots, one of them farmed continuously with barley, soybean and barley in a no-tillage system for more than 10 years, with $\mathrm{pH} 6.0$ and fertilized with $P$ and $K$. A second plot was maintained undisturbed, with native vegetation (mostly grasses), with pH 4.8 and low soil fertility. The study was carried out in a Typical Red Dystrophic Argisol at an experimental station of Universidade Federal do Rio Grande do Sul, Eldorado do Sul - RS, in 2002 and 2003. Seasonal soil samples were taken on average every ten days at the farmed plot (5/31/2002 until 10/23/2003) and fallow plot (6/13/2002 until 10/17/ 2002). Short-term samples (5/19/2003 until 09/29/2003) were taken every one to three days. In both cases, soil samples were taken from the top $20 \mathrm{~cm}$ soil layer. No nitrogen fertilization was applied in either plot during the whole period. Seasonal trends showed increasing mineral $\mathrm{N}\left(\mathrm{NH}_{4}^{+}\right.$and $\left.\mathrm{NO}_{3}{ }^{-}\right)$from spring to summer, and decreasing from autumn to winter, in 2002 and 2003. $N$ mineralization followed approximately the average temperature oscillation pattern for the sampled period. Mineral $\mathrm{N}$ in the short-term samples was not constant from one day to the next. The variation was related to rainfall occurrence, which was responsible for a sharp decrease in mineral N content. Nitrate and ammonium contents in the undisturbed plot were similar to those observed in the plot growing barley. The 1:1 ratio of $\mathrm{NH}_{4}{ }^{+}$and $\mathrm{NO}_{3}{ }^{-}$observed both in the farmed plot and the undisturbed plot indicates that there is a balance between both ionic forms, independent of soil $\mathrm{pH}$ and fertility level.
\end{abstract}

Index terms: nitrogen, non-tillage system, temperature, precipitation, $p H$.

\section{INTRODUÇÃO}

O N de compostos orgânicos no solo encontra-se, em grande parte, em formas praticamente indisponíveis às plantas. A fração orgânica, de $0,1 \mathrm{a}$ $50 \%$ do $\mathrm{N}$ total do solo, está sujeita à ação microbiana (Barber, 1995) e é, constantemente, convertida em fração mineral $\left(\mathrm{NH}_{4}{ }^{+}, \mathrm{NO}_{3}{ }^{-}\right.$e $\left.\mathrm{NO}_{2}{ }^{-}\right)$pelo processo de mineralização.

Os íons $\mathrm{NH}_{4}{ }^{+} \mathrm{e} \mathrm{NO}_{3}{ }^{-}$são as formas predominantes de $\mathrm{N}$ mineral disponíveis às plantas, e a concentração desses íons ao longo do perfil do solo é muito variável. Nas camadas superficiais de alguns solos, aproximadamente $5 \%$ do $\mathrm{N}$ total está sob a forma de $\mathrm{NH}_{4}{ }^{+}$, e em camadas subsuperficiais a quantidade de $\mathrm{N}$ amoniacal pode atingir valores de até $50 \%$ do total de N (Walsh \& Murdock, 1960; Keeney \& Bremner, 1964).

As concentrações médias de $\mathrm{NH}_{4}{ }^{+}$encontradas em solos agrícolas são, geralmente, $10-1000$ vezes menores que as de $\mathrm{NO}_{3}{ }^{-}$(entre 0,05 e $0,2 \mathrm{mmol} \mathrm{L}^{-1}$ de $\mathrm{NH}_{4}{ }^{+}$ em alguns solos) (Marschner, 1995; Owen \& Jones, 2001), devido, principalmente, a limitações em sua difusão (Schjoerring et al., 2002) e à rápida oxidação do $\mathrm{NH}_{4}{ }^{+}$para $\mathrm{NO}_{3}^{-}$pelas bactérias nitrificadoras (Norton, 2000). No entanto, podem-se encontrar valores que variam entre 2 e até mais de $20 \mathrm{mmol} \mathrm{L}^{-1}$ de $\mathrm{NH}_{4}{ }^{+}$(Crawford \& Glass, 1998). Em florestas permanentes e ecossistemas com pastagens naturais, o $\mathrm{NH}_{4}{ }^{+}$é a forma predominante de $\mathrm{N}$ disponível às plantas (Pearson \& Stewart, 1993; Bijlsma et al., 2000; Schjoerring et al., 2002). $\mathrm{O} \mathrm{NO}_{3}{ }^{-}$é a forma de $\mathrm{N}$ que prevalece em solos aerados com $\mathrm{pH}$ acima de 4,0 (Armstrong, 1982), sendo a sua concentração variável de 1 a 5 mmol L-1 (Owen \& Jones, 2001).

A disponibilidade, a localização e formas de $\mathrm{N}$ encontradas no perfil do solo podem variar em decorrência das alterações nas taxas de amonificação, nitrificação e desnitrificação e das características dos íons $\mathrm{NH}_{4}^{+}$e $\mathrm{NO}_{3}^{-}$(Miller \& Cramer, 2004). As taxas de amonificação, nitrificação e desnitrificação são regidas por fatores que interferem diretamente na atividade microbiana, como $\mathrm{pH}$, temperatura, aeração e umidade do solo (Lewis, 1986). Na solução do solo, a predominância do $\mathrm{NH}_{4}{ }^{+}$em relação ao $\mathrm{NO}_{3}{ }^{-}$é observada, em geral, em ambientes com baixo $\mathrm{pH}$, baixa temperatura e alta umidade, com acúmulo de compostos fenólicos alelopáticos e anaerobiose (Djik \& Eck, 1995) que inibem a nitrificação (Stark \& Hart, 1997). A taxa de nitrificação é próxima de zero em solos com excesso d'água e em solos com temperaturas inferiores a $5{ }^{\circ} \mathrm{C}$ ou superiores a $40{ }^{\circ} \mathrm{C}$ (Lewis, 1986).

$\mathrm{O}$ íon $\mathrm{NO}_{3}{ }^{-}$é o último elemento formado no ciclo biológico do $\mathrm{N}$ no solo e é a forma preferencial de absorção de $\mathrm{N}$ pela maioria das plantas cultivadas. Ele é pouco retido no solo, principalmente devido à sua baixa energia de ligação aos argilominerias e à matéria orgânica (Reisenauer, 1978), podendo ser, assim, lixiviado para as camadas subsuperficiais do perfil. $\mathrm{O}$ íon $\mathrm{NH}_{4}^{+}$, por ser um cátion, não é facilmente 
lixiviado e, quando mineralizado, permanece no complexo trocável do solo, podendo ser absorvido pelas plantas ou oxidado para $\mathrm{NO}_{3}{ }^{-}$.

Assim, os valores de $\mathrm{N}$ mineral no solo podem variar entre regiões e dentro de uma mesma região, entre estações de crescimento durante o ciclo de desenvolvimento das culturas. Nesse contexto, este trabalho avaliou a variação dos teores de $\mathrm{NH}_{4}{ }^{+} \mathrm{e} \mathrm{NO}_{3}{ }^{-}$ ao longo de um ano (variação entre estações) e flutuações diárias numa mesma estação em áreas de cultivo de grãos e de vegetação nativa (campo limpo) em um Argissolo Vermelho distrófico típico.

\section{MATERIAL E MÉTODOS}

O estudo foi realizado na Estação Experimental Agronômica da UFRGS (EEA), Eldorado do Sul-RS, nos anos agrícolas de 2002 e de 2003. A EEA está localizada na região da Depressão Central do Estado, com altitude máxima de $46 \mathrm{~m}$ acima do nível do mar. O solo é classificado como Argissolo Vermelho distrófico típico pertencente à unidade de mapeamento São Jerônimo (Embrapa, 1999).

O clima da região é do tipo Cfa, de acordo com a classificação climática de Köppen, correspondente ao "subtropical de verão úmido quente" (Ipagro, 1979). A média anual de precipitação pluvial é de $1.440 \mathrm{~mm}$ e as temperaturas do ar médias mensais variam entre 13,9 e $24,9{ }^{\circ} \mathrm{C}$ nos meses mais frio e mais quente, respectivamente (Bergamaschi \& Guadagnin, 1990).

Os trabalhos foram realizados em dois locais adjacentes: (a) área cultivada em que se estabeleceram os cultivos da cevada no inverno de 2002 (sobre resteva de milho) e, logo após, da soja (sobre a resteva de cevada) e, subseqüentemente, da cevada (na resteva da soja, no inverno de 2003); a área se encontra no sistema plantio direto há mais de 10 anos e os cultivos foram feitos sem a adição de adubo nitrogenado; e (b) áreas não-cultivadas, cobertas com vegetação nativa (campo limpo com predominância de gramíneas).

A análise de solo da área cultivada foi realizada em amostras retiradas entre as profundidades de 0 $0,2 \mathrm{~m}$ antes da semeadura da cevada, em maio de 2002 , e teve o seguinte resultado: $\mathrm{pH}$ em água: 6,0; $\mathrm{P}$ : 3,1 $\mathrm{mg} \mathrm{dm}^{-3}$ (Mehlich-1); K: $164 \mathrm{mg} \mathrm{dm}^{-3}$ (Mehlich-1); $\mathrm{Al}^{3+}: 0 \mathrm{cmol}_{\mathrm{c}} \mathrm{dm}^{-3}$ (extração com KCl $1 \mathrm{~mol} \mathrm{~L}^{-1}$ ); $\mathrm{Ca}^{2+}$ : $3,3 \mathrm{cmol}_{\mathrm{c}} \mathrm{dm}^{-3}$ (extração com $\mathrm{KCl} 1 \mathrm{~mol} \mathrm{~L}^{-1}$ ); $\mathrm{Mg}^{2+}$ : $1,9 \mathrm{cmol}_{\mathrm{c}} \mathrm{dm}^{-3}$ (extração com KCl $1 \mathrm{~mol} \mathrm{~L}^{-1}$ ); matéria orgânica: $24 \mathrm{~g} \mathrm{~kg}^{-1}$ (digestão úmida); saturação de bases: $84 \%$. A semeadura das culturas de cevada e de soja foi realizada em sistema de plantio direto em profundidade média de $3,0 \mathrm{~cm}$. Antes da semeadura de cada uma das culturas, procedeu-se à aplicação de $300 \mathrm{~kg} \mathrm{ha}^{-1}$ de superfosfato triplo + cloreto de potássio com fórmula 0-21-30. O controle de plantas daninhas e os tratamentos fitossanitários, inclusive o tratamento de semente, foram realizados de acordo com a recomendação para cada cultura. A semeadura e a colheita foram efetuadas mecanicamente.

A análise de solo da área não-cultivada mostrou o seguinte resultado: $\mathrm{pH}$ em água: 4,$8 ; \mathrm{P}: 2,1 \mathrm{mg} \mathrm{dm}^{-3}$ (Mehlich-1); K: $128 \mathrm{mg} \mathrm{dm}^{-3}$ (Mehlich-1); $\mathrm{Al}^{3+}$ : $0,6 \mathrm{cmol}_{\mathrm{C}} \mathrm{dm}^{-3}$ (extração com KCl $1 \mathrm{~mol} \mathrm{~L}^{-1}$ ); $\mathrm{Ca}^{2+}$ : $1,8 \mathrm{cmol}_{\mathrm{c}} \mathrm{dm}^{-3}$ (extração com KCl $1 \mathrm{~mol} \mathrm{~L}^{-1}$ ); $\mathrm{Mg}^{2+}$ : $1,2 \mathrm{cmol}_{\mathrm{c}} \mathrm{dm}^{-3}$ (extração com KCl $1 \mathrm{~mol} \mathrm{~L}^{-1}$ ); matéria orgânica: $22 \mathrm{~g} \mathrm{~kg}^{-1}$ (digestão úmida); saturação por bases: 38 \%. Nesta área, não foram efetuadas correção do solo e adição de fertilizantes.

As amostragens de solo de caráter estacional na área cultivada foram iniciadas em 31/05/2002 e estenderam-se até 23/10/2003. As amostragens de solo foram feitas, em média, a cada dez dias ao longo dos ciclos das culturas de cevada e soja. As amostragens de solo de caráter estacional na área mantida sob vegetação nativa (área não-cultivada) foram iniciadas em 13/06/2002 e estenderam-se até 17/10/2002. Nesta área, as avaliações de caráter diário foram feitas ao longo do ciclo da cevada durante o outono/inverno de 2003 (16/05 a 30/09 de 2003), com amostragens realizadas em intervalos de um a três dias.

Nos dois tipos de avaliações, as amostras foram retiradas aleatoriamente na camada superficial do solo até a profundidade de 0,2 $\mathrm{m}$ utilizando-se trado holandês, tanto na área cultivada quanto na área mantida sob vegetação nativa. A metodologia de amostragem de solo na área cultivada consistiu inicialmente na retirada dos resíduos culturais nas entrelinhas da cultura instalada e, posteriormente, procedeu-se à coleta de solo. Na área não-cultivada, nos pontos de amostragem de solo, foi retirada a vegetação superficial e, posteriormente, realizou-se a coleta de solo. Nas duas áreas, foram retiradas três subamostras que compunham cada amostra de solo. As amostras (em número de três por área) coletadas no campo foram imediatamente colocadas em solução salina de $\mathrm{KCl} 2 \mathrm{~mol} \mathrm{~L}^{-1}$ e levadas ao laboratório para análise. A determinação dos teores de $\mathrm{NH}_{4}{ }^{+}{\mathrm{e} \mathrm{NO}_{3}}^{-}$ seguiu a metodologia utilizada para a avaliação do $\mathrm{N}$ mineral descrita por Tedesco (1995). Os dados de temperatura do ar e precipitação pluviométrica, para o período avaliado, foram registrados na Estação Meteorológica do Departamento de Forrageiras e Agrometeorologia, distante $2 \mathrm{~km}$ do local do trabalho. Os dados experimentais não foram submetidos à análise estatística, pois apenas descrevem o comportamento dos teores de $\mathrm{N}$ mineral no solo ao longo de estações de crescimento em áreas com manejo diferenciado.

\section{RESULTADOS E DISCUSSÃO}

A disponibilidade de $\mathrm{N}$ mineral na área cultivada, ao longo do período experimental, foi baixa, resultando em valores médios de $10 \mathrm{mg} \mathrm{kg}^{-1}$ tanto para $\mathrm{NO}_{3}{ }^{-}$ 
como para $\mathrm{NH}_{4}{ }^{+}$(Figura 1), possivelmente devido ao baixo teor de matéria orgânica observado na área cultivada (24 $\left.\mathrm{g} \mathrm{kg}^{-1}\right)$ e das condições de cultivo, associados a condições de temperatura e umidade do solo. Os valores de $\mathrm{N}$ mineral encontrados foram baixos quando comparados aos obtidos em outros estudos em áreas agricultáveis (Lamothe, 1994; Kurokawa et al., 2005).

A avaliação estacional, em intervalos de dez dias, no ano agrícola de 2002, mostrou valores de $\mathrm{NH}_{4}{ }^{+} \mathrm{e}$ de $\mathrm{NO}_{3}^{-}$que oscilaram entre 0 e $5 \mathrm{mg} \mathrm{kg}^{-1}$ (agosto e setembro) e pouco acima de $10 \mathrm{mg} \mathrm{kg}^{-1}$, em dezembro (Figura 1). Já no ano de 2003, as coletas indicaram variações entre $15 \mathrm{mg} \mathrm{kg}^{-1}$ (janeiro) e menos que $5 \mathrm{mg} \mathrm{kg}^{-1}$ (junho). Esses resultados permitiram identificar os picos de máximo e mínimo dos valores de $\mathrm{NH}_{4}{ }^{+} \mathrm{e} \mathrm{NO}_{3}{ }^{-}$entre estações. Diversos trabalhos avaliando o comportamento do $\mathrm{N}$ mineral ao longo do tempo (Watson \& Poland, 1999; Espindola et al., 2001; Kurokawa et al., 2005) evidenciaram a influência ambiental de cada estação sobre a mineralização do N.

Nos meses de primavera, o $\mathrm{N}$ mineral $\left(\mathrm{NH}_{4}{ }^{+} \mathrm{e}\right.$ $\mathrm{NO}_{3}{ }^{-}$) teve aumento gradual, com valores acima dos obtidos no período de inverno, tanto em 2002 quanto em 2003, evidenciando o incremento da mineralização do N nesta estação (Figura 1). Os maiores e menores valores de $\mathrm{N}$ mineral foram constatados no verão e inverno, respectivamente. As variações estacionais acompanharam, aproximadamente, as oscilações na temperatura do mesmo período (Figura 1), coincidindo, no inverno, com as mais baixas temperaturas e, no verão, com os maiores valores de temperaturas médias.
A oscilação do $\mathrm{N}$ mineral ocorre devido ao processo biológico de mineralização do $\mathrm{N}$ orgânico, o qual envolve a ação de microrganismos amonificadores e nitrificadores. A magnitude do processo depende de fatores como temperatura e umidade do solo (Campbell \& Biederbeck, 1972; Dalias et al., 2002). $\mathrm{O}$ aumento progressivo nos valores de $\mathrm{NH}_{4}{ }^{+}$e $\mathrm{NO}_{3}{ }^{-}$, observado na primavera e sua manutenção no verão, possivelmente deveu-se à elevação na temperatura (acima de $20^{\circ} \mathrm{C}$ ) durante essas duas estações, estimulando a atividade microbiana. $\mathrm{O}$ aumento nas taxas de mineralização do $\mathrm{N}$ em solo incubado ocorre quando a temperatura passa de 15 para $35^{\circ} \mathrm{C}$ (Stanford \& Smith, 1972; Stark \& Firestone, 1996; Nicolardot et al., 2001), sendo a faixa de temperatura mais favorável para o processo de nitrificação situada entre 26 e $32{ }^{\circ} \mathrm{C}$ (Moreira \& Siqueira, 2002). Durante os meses de inverno, com baixas temperaturas, as condições são desfavoráveis para a sobrevivência da microbiota no solo (Wong \& Nortcliff, 1995) e, portanto, para a mineralização do $\mathrm{N}$ orgânico.

No período amostrado (outono de 2002 à primavera de 2003), na maioria das amostras da área cultivada, ocorreu semelhança nos valores de $\mathrm{N}$ na forma amoniacal e na forma nítrica, sendo os valores estacionais médios das concentrações de $\mathrm{NH}_{4}{ }^{+} \mathrm{e} \mathrm{NO}_{3}{ }^{-}$, respectivamente, iguais a 6,89 e $7,42 \mathrm{mg} \mathrm{kg}^{-1}$ (outono e inverno de 2002); 5,55 e 6,72 $\mathrm{mg} \mathrm{kg}^{-1}$ (outono e inverno de 2003); 14,89 e 12,56 mg kg-1 (primavera e verão de 2002). Isso pode estar relacionado a diversas causas, como a preferência de absorção pelo $\mathrm{NO}_{3}{ }^{-} \mathrm{em}$ detrimento do $\mathrm{NH}_{4}{ }^{+}$pelas espécies cultivadas no

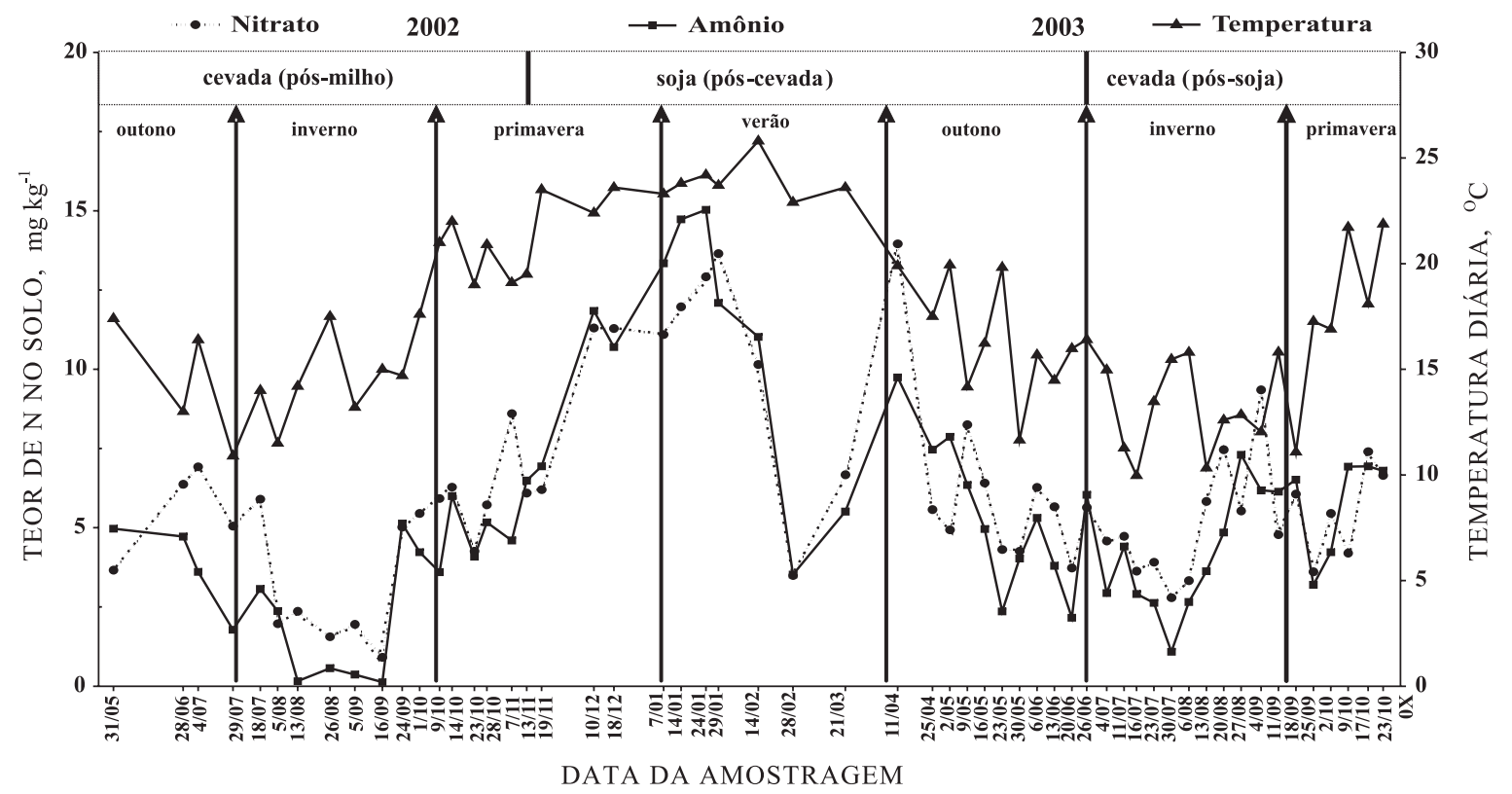

Figura 1. Temperatura média e variação estacional dos teores de amônio $\left(\mathrm{NH}_{4}^{+}\right)$e nitrato $\left(\mathrm{NO}_{3}{ }^{-}\right)$nas amostragens de solo, no período de 31/05/2002 a 23/10/2003, em área cultivada com cevada (sob resteva de milho - inverno de 2002), soja (sob resteva de cevada - verão 2002/ 2003) e cevada (sob resteva de soja - inverno 2003), Eldorado do Sul-RS. 
experimento. A soja e a cevada, em solos aerados, utilizam o $\mathrm{NO}_{3}{ }^{-}$como principal fonte de $\mathrm{N}$ (Britto \& Kronzucker, 2002; Schjoerring et al., 2002). Outras causas podem ser a predominância de bactérias amonificadoras sobre nitrificadoras; o baixo conteúdo de $\mathrm{O}_{2}$ resultando em menor produção de $\mathrm{NO}_{3}{ }^{-}$pelo processo de nitrificação (durante o período avaliado); e perdas desse íon por lixiviação e, ou, desnitrificação. A temperatura e a umidade do solo são fatores reguladores para as bactérias nitrificadoras (Paul \& Clark, 1996). Em geral, a quantidade de bactérias nitrificadoras no solo não é alterada em curtos períodos (Woldendorp \& Laanbroek, 1989), contribuindo para a predominância da forma nítrica no solo. No entanto, neste trabalho, observou-se que os teores de $\mathrm{NH}_{4}{ }^{+} \mathrm{em}$ algumas amostragens superaram os de $\mathrm{NO}_{3}^{-}$. O acúmulo de $\mathrm{NH}_{4}{ }^{+}$em determinadas microrregiões no solo pode ser decorrente da redução na taxa de nitrificação ocasionado pelo baixo suprimento de $\mathrm{O}_{2}$ (Cantarella, 2007). Situação semelhante pode ter ocorrido neste estudo, em que o baixo suprimento de $\mathrm{O}_{2}$ ocasionado, principalmente, pela alta umidade do solo (após elevadas precipitações) contribuiu para o decréscimo dos teores de $\mathrm{NO}_{3}{ }^{-}$em algumas amostragens pontuais.

A associação da quantidade e distribuição estacional da precipitação com as oscilações dos teores de $\mathrm{N}$ mineral determinados em intervalos de dez dias não foi verificada com precisão no trabalho (Figura 2). $\mathrm{Na}$ região onde foi desenvolvido o trabalho, a precipitação pluvial é relativamente bem distribuída ao longo do ano, sem uma estação seca bem definida. Neste contexto, embora a água seja fundamental no processo de mineralização, as chuvas podem ser o fator que explica as flutuações estacionais nos teores de $\mathrm{N}$ mineral.
As avaliações diárias, realizadas no inverno de 2002 (entre maio e setembro) (Figura 3) mostraram valores de $\mathrm{N}$ mineral variando entre 3,33 e 19,76 $\mathrm{mg} \mathrm{kg}^{-1}$, com valores médios em torno de $9,48 \mathrm{mg} \mathrm{kg}^{-1}$. Em intervalos de um a três dias, não houve correspondência entre oscilações na temperatura e nos teores de $\mathrm{N}$ mineral (Figura 3). A precipitação pluvial observada ao longo do período avaliado (806 $\mathrm{mm}$ em 137 dias) pode ter contribuído tanto para a lixiviação do $\mathrm{NO}_{3}{ }^{-}$ quanto para a formação de sítios transitórios de anaerobiose, que impedem a nitrificação e ocasionam a queda nos teores de $\mathrm{N}$ mineral (Figura 4). Alguns ânions, quando não absorvidos pelas plantas, estão sujeitos à lixiviação para camadas subsuperficiais do solo (Costa et al., 1999). Esse é o caso do $\mathrm{NO}_{3}{ }^{-}$que possui alta mobilidade e, por isso, é facilmente percolado com a água no perfil do solo (Edwards et al.,1972). A alta mobilidade do $\mathrm{NO}_{3}{ }^{-}$no solo é devida, principalmente, ao alto coeficiente de difusão, cem vezes maior que os de outros íons como o $\mathrm{NH}_{4}{ }^{+}$(Owen \& Jones, 2001). Restrições na disponibilidade de $\mathrm{O}_{2}$, além de ocasionar redução na taxa de nitrificação, podem favorecer a desnitrificação, promovendo decréscimo nos teores de $\mathrm{NO}_{3}{ }^{-}$(Cantarella, 2007) e, conseqüentemente, redução no teor de $\mathrm{N}$ mineral total no solo (Reisenauer, 1978). Perdas de N nas formas de $\mathrm{N}_{2}$ e $\mathrm{N}_{2} \mathrm{O}$ ocorrem em solos em condições aeróbias, visto que mesmo nesses solos evidenciam-se sítios anaeróbios (Sexstone et al., 1985), decorrentes de fatores como compactação e alta umidade do solo.

A ocorrência das chuvas ocasionou as maiores variações nos teores de $\mathrm{N}$ mineral, quando estes foram avaliados em intervalos de um a três dias (Figura 4). Após a ocorrência da precipitação pluvial, houve uma queda nos teores de $\mathrm{N}$ mineral, os quais retornavam,

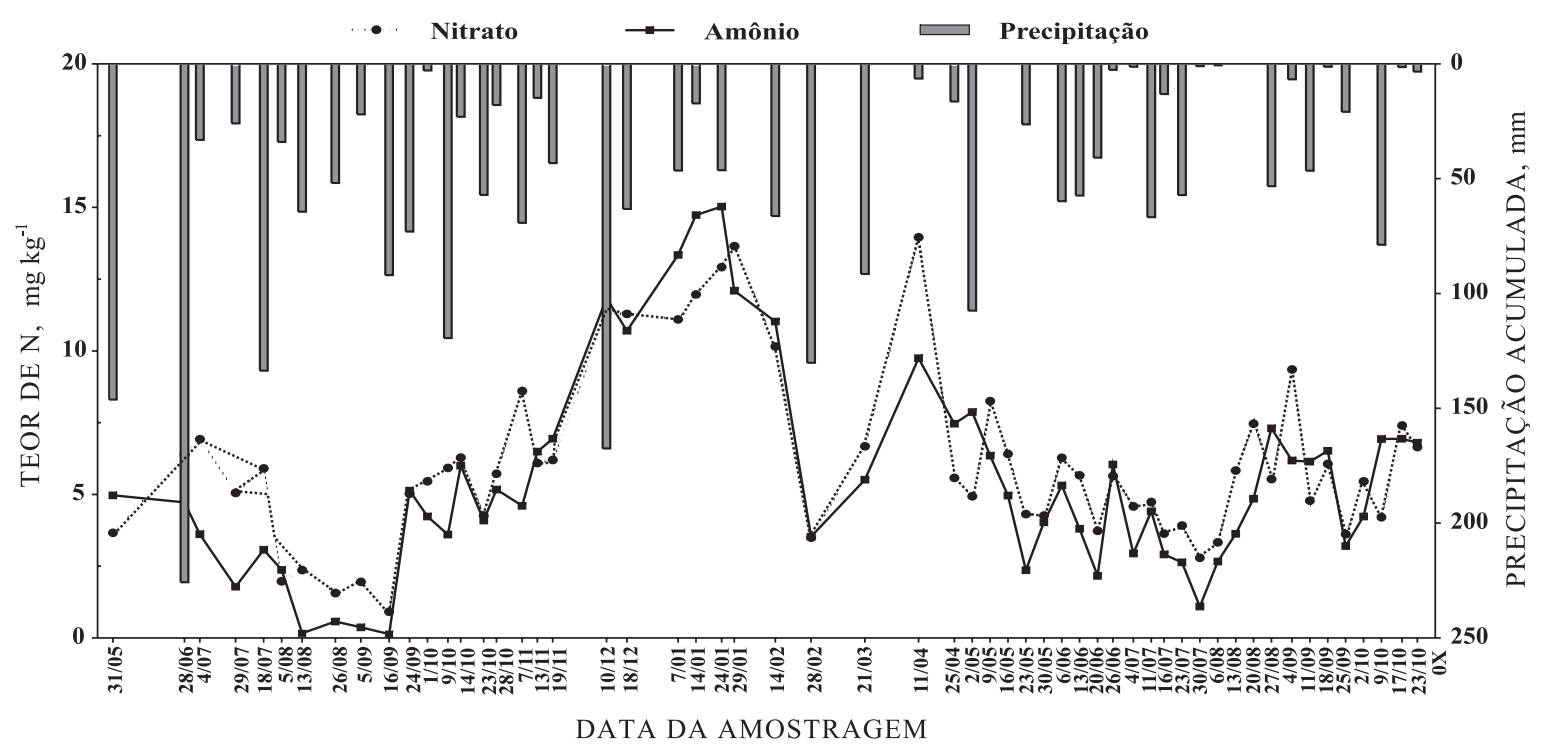

Figura 2. Precipitação pluvial acumulada e variação estacional dos teores de amônio $\left(\mathrm{NH}_{4}^{+}\right)$e nitrato $\left(\mathrm{NO}_{3}{ }^{-}\right)$ nas amostragens de solo, no período de 31/05/2002 a 23/10/2003, em área cultivada com cevada (sob resteva de milho - inverno de 2002), soja (sob resteva de cevada - verão 2002/ 2003) e cevada (sob resteva de soja - inverno 2003), Eldorado do Sul-RS. 


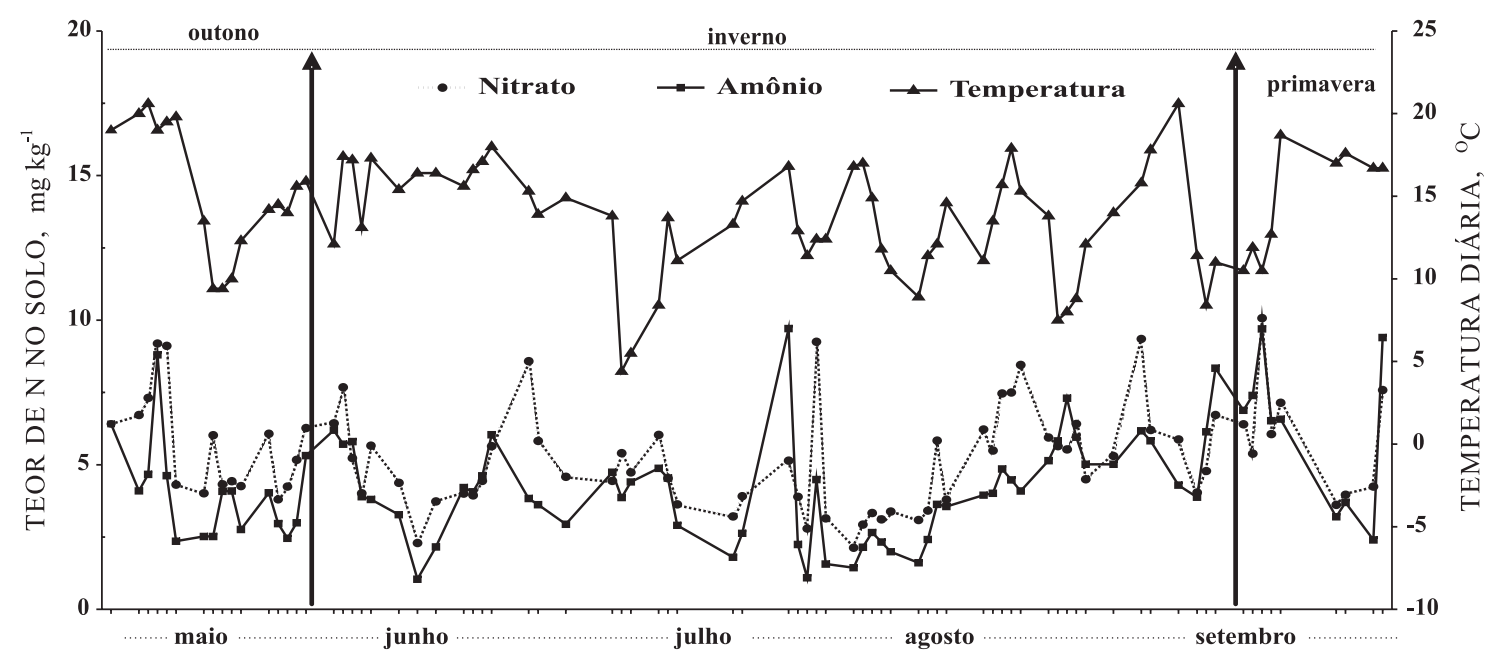

DATA DA AMOSTRAGEM

Figura 3. Temperatura média e variação diária dos teores de amônio $\left(\mathrm{NH}_{4}^{+}\right)$e nitrato $\left(\mathrm{NO}_{3}{ }^{-}\right)$nas amostragens de solo, no período de 16/05/2003 a 30/09/2003, em área cultivada com cevada (sob resteva de soja), Eldorado do Sul, RS.

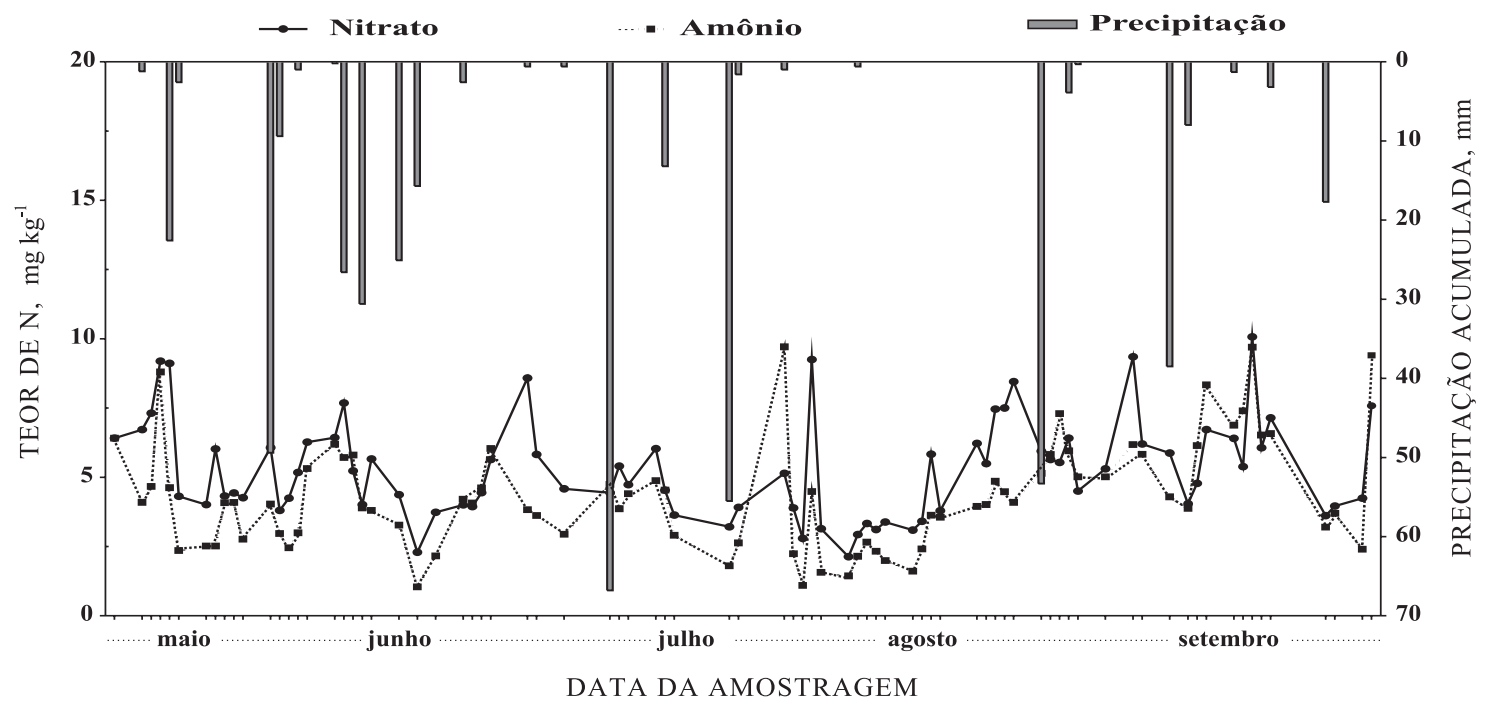

Figura 4. Precipitação acumulada e variação diária dos teores de amônio $\left(\mathrm{NH}_{4}^{+}\right)$e nitrato $\left(\mathrm{NO}_{3}{ }^{-}\right)$nas amostragens de solo, no período de 16/05/2003 a 30/09/2003, em área cultivada com cevada (sob resteva de soja), Eldorado do Sul-RS.

em um pequeno número de dias, aos seus valores originais médios, característicos da faixa determinada pela temperatura de cada estação de crescimento (Figura 4).

$\mathrm{Na}$ área sob vegetação nativa, o $\mathrm{N}$ mineral $\left(\mathrm{NH}_{4}{ }^{+}\right.$ e $\mathrm{NO}_{3}{ }^{-}$) teve comportamento semelhante ao observado na área cultivada com cevada (Figura 5). A proporção de aproximadamente 1:1 entre os teores de $\mathrm{NH}_{4}{ }^{+} \mathrm{e}$ $\mathrm{NO}_{3}{ }^{-}$também foi observada na área mantida sobre vegetação nativa. Estudos em áreas com pastagem natural mostraram que os teores de $\mathrm{NH}_{4}{ }^{+}$foram superiores aos de $\mathrm{NO}_{3}{ }^{-}$(Munro, 1966; Moore \& Waid, 1971; Watson \& Poland, 1999). Estas áreas, em geral, possuem baixa fertilidade natural e baixo $\mathrm{pH}$, constatados também nos solos deste estudo, desfavorecendo a população de microrganismos nitrificadores (Bijlsma et al., 2000; Schjoerring et al., 2002), promovendo, assim, o acúmulo de $\mathrm{NH}_{4}{ }^{+}$. Neste caso, a semelhança nas proporções dos íons $\mathrm{NH}_{4}{ }^{+} \mathrm{e}$ $\mathrm{NO}_{3}{ }^{-}$encontrados na área com vegetação nativa e na área cultivada sugere que pode estar ocorrendo redução do $\mathrm{NO}_{3}{ }^{-}$no solo. As formas oxidadas de $\mathrm{N}$, como o $\mathrm{NO}_{3}{ }^{-}$, podem ser reduzidas por processos assimilatório e dissimilatório (Moreira \& Siqueira, 2002). Dentre eles, a redução assimilatória pela qual os microrganismos do solo incorporam o $\mathrm{NO}_{3}^{-}$às suas células 

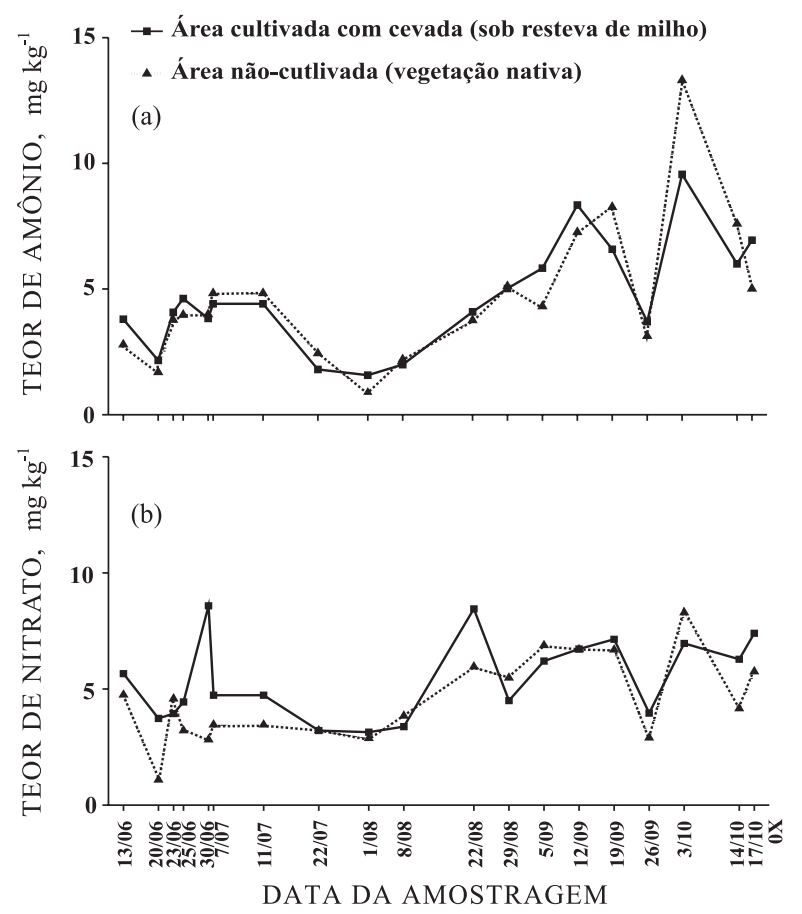

Figura 5. Teores de amônio (a) e nitrato (b) do solo em amostras retiradas da área cultivada com cevada (sob resteva de milho) e da área nãocultivada (vegetação nativa) no período entre 13/06/2002 e 17/10/2002, Eldorado do Sul-RS.

(Cantarella, 2007), a redução dissimilatória do $\mathrm{NO}_{3}{ }^{-}$ para $\mathrm{NH}_{4}^{+}$, e a desnitrificação do $\mathrm{NO}_{3}{ }^{-}$para $\mathrm{N}_{2}$ e $\mathrm{N}_{2} \mathrm{O}$, reguladas pela disponibilidade de $\mathrm{O}_{2}$ (Moreira \& Siqueira, 2002), podem ocorrer em solos agrícolas.

Além das variáveis ambientais, como temperatura e precipitação pluvial, avaliadas neste estudo, é provável que os atributos físicos e químicos do solo também estejam exercendo efeito sobre a atividade dos microrganismos amonificadores e nitrificadores, como já relatado em estudos semelhantes (Jarvis \& Barraclough, 1991; Espindola et al., 2001; Dalias et al., 2002).

\section{CONCLUSÕES}

1. Os teores de $\mathrm{N}$ mineral variaram sazonalmente, acompanhando as oscilações na temperatura média.

2. O regime de precipitação pluvial foi o fator mais importante para diferenciar as oscilações de curto prazo do $\mathrm{N}$ mineral dentro de uma mesma estação, sendo pouco importante sobre as oscilações sazonais nos teores de $\mathrm{N}$ mineral.

3. A proporção 1:1 dos íons $\mathrm{NH}_{4}{ }^{+}$e $\mathrm{NO}_{3}{ }^{-}$indica que o sistema plantio direto não alterou a proporção observada no sistema sob vegetação nativa.

\section{LITERATURA CITADA}

ARMSTRONG, W. Waterlogged soils. In: ETHERINGTON, J.R. Environment and plant ecology. 2.ed. London, John Wiley \& Sons, 1982. p.290-332.

BARBER, S.A. Soil nutrient bioavailability: A mechanistic approach. 2.ed. New York, Wiley-Interscience, 1995. 384p.

BERGAMASCHI, H. \& GUADAGNIN, M.R. Agroclima da Estação Experimental Agronômica - UFRGS. Porto Alegre, Universidade Federal do Rio Grande do Sul, 1990. $60 \mathrm{p}$.

BIJLSMA, R.J.; LAMBERS, H. \& KOOIJMAN, S. A dynamic wholeplant model of integrated metabolism of nitrogen and carbon. 1. Comparative ecological implications of ammonium-nitrate interactions. Plant Soil, 220:49-69, 2000 .

BRITTO, D.T. \& KRONZUCKER, H.J. $\mathrm{NH}_{4}{ }^{+}$toxicity in higher plants: A critical review. J. Plant Physiol., 159:567-584, 2002.

CAMPBELL, C.A. \& BIEDERBECK, V.O. Influence of fluctuating temperatures and constant soil moistures on nitrogen changes in amended and unamended loam. Can. J. Soil Sci. 52:323-336, 1972.

CANTARELLA, H. Nitrogênio. In: NOVAIS, R.F.; ALVAREZ V., V.H.; BARROS, N.F.; FONTES, R.L.F.; CANTARUTTI, R.B. \& NEVES, J.C.L., eds. Fertilidade do solo. Viçosa, MG, Sociedade Brasileira de Ciência do Solo, 2007. p.375470 .

COSTA, S.N.; MARTINEZ, M.A.; MATOS, A.T. \& RAMOS, V.B.N. Mobilidade de nitrato em colunas de solo sob condições de escoamento não permanente. R. Bras. Eng. Agric. Amb., 3:190-194, 1999 .

CRAWFORD, N.M. \& GLASS, A.D.M. Molecular and physiological aspects of nitrate uptake in plants. Trends Plant Sci., 3:389-395, 1998.

DALIAS, P.; ANDERSON, J.M.; BOTTNER, P. \& COÛTEAUX, M.M. Temperature responses of net $\mathrm{N}$ mineralization and nitrification in conifer forest soils incubated under standard laboratory conditions. Soil Biol. Biochem., 34:691-701, 2002.

DJIK, E. \& ECK, N. Ammonium toxicity and nitrate response of axenically grown dactylorhiza-incarnata seedlings. New Phytol., 131:361-367, 1995.

EDWARDS, D.M.; FISCHBACH, P.E. \& YOUNG, L.L. Movement of nitrate under irrigated agriculture. Trans. Am. Soc. Agric. Eng., 15: 73-75, 1972.

EMPRESA BRASILEIRA DE PESQUISA AGROPECUÁRIA EMBRAPA. Centro Nacional de Pesquisa de Solos. Manual de métodos de análises de solos. 2.ed. Rio de Janeiro, 1999. 212p.

ESPÍNDOLA, J.A.A.; ALMEIDA, D.; GUERRA, J.G.M. \& SILVA, E.M.R. Flutuação sazonal da biomassa microbiana e teores de nitrato e amônio de solo coberto com Paspalum notatum em um agroecossistema. Floresta Amb., 8:104113, 2001. 
IPAGRO. Observações meteorológicas no Estado do Rio Grande do Sul. Porto Alegre, 1979. 272p. (Boletim Técnico, 3)

JARVIS, S.C. \& BARRACLOUGH, D. Variation in mineral nitrogen under grazed grassland swards. Plant Soil, 138:177-188, 1991

KEENEY, D.R. \& BREMNER, J.M. Effect of cultivation on the nitrogen distribution in soils. Soil Sci. Soc. Am. Proc., 28:653-656, 1964.

KUROKAWA, Y. Nitrogen balance and seasonal fluctuations in soil nitrogen contents in a corn (Zea maysL)-rye (Secale cerealeL) rotation field. Grass. Sci., 51:79-54, 2005.

LAMOTHE, A.G. Manejo del nitrógeno para aumentar productividad en trigo. Montevideo, INIA, 1994. 26p. (Série Técnica, 54)

LEWIS, O.A.M. Plants and nitrogen. London, Cambridge Press, 1986. $100 \mathrm{p}$

MARSCHNER, H. Mineral nutrition of higher plants. 2.ed. London, Academic Press, 1995. 889p.

MILLER, A.J. \& CRAMER, M.D. Root nitrogen acquisition and assimilation. Plant Soil, 274:1-36, 2004.

MOORE, D.R.E. \& WAID, J.S. The influence of washings of living roots on nitrification. Soil Biol. Biochem., 3:69-83, 1971.

MOREIRA, F.M.S. \& SIQUEIRA, J.O. Microbiologia e bioquímica do solo. Lavras, Universidade Federal de Lavras, 2002. 625p.

MUNRO P.E. Inhibition of nitrifiers by grass root extracts. J. Appl. Ecol., 3:231-238, 1966.

NICOLARDOT, B.; RECOUS, S. \& MARY, B. Simulation of C and $\mathrm{N}$ mineralization during crop residue decomposition: A simple dynamics model based on the $\mathrm{C}: \mathrm{N}$ ratio of the residue. Plant Soil, 228:83-103, 2001.

NORTON, J.M. Nitrification. In: SUMMER, M.E., ed. Handbook of soil science. Boca Raton, CRC Press, 2000. p.C160-C181.

OWEN, A.G. \& JONES, D.L. Competition for amino acids between wheat roots and rhizosphere microorganisms and the role of amino acids in plant $\mathrm{N}$ acquisition. Soil Biol. Biochem., 33:651-657, 2001.

PAUL, E.A. \& CLARK, F.E. Soil microbiology and biochemistry. San Diego, Academic Press, 1996. 188p.
PEARSON, J. \& STEWART, G.R. The deposition of atmospheric ammonia and its effects on plants. New Phytol., 125:283-305, 1993.

REISENAUER, H.M. Absorption and utilization of ammonium nitrogen by plants. In: NIELSEN, D.R. \& McDONALD, J.G. Nitrogen in the environment. London, Academic Press, 1978. v.2. p.157-170.

SCHJOERRING, J.K.; HUSTED, S.; MACK, G. \& MATTSSON, M. The regulation of ammonium translocation in plants. J. Exper. Bot., 53:883-890, 2002.

SEXSTONE, A.J.; REVSBECH, N.P.; PARKIN, T.B. \& TIEDJE, J.M. Direct measurement of oxygen profiles and denitrification rates in soil aggregates. Soil Sci. Soc. Am. J., 49:645-651, 1985.

STANFORD, G. \& SMITH, S.J. Nitrogen mineralization potential of soils. Soil Sci. Soc. Am. J. 36:465-472, 1972.

STARK, J.M. \& FIRESTONE, M.K. Kinetic characteristics of ammoniumoxidizer communities in a California oak woodland-annual grassland. Soil Biol. Biochem., 28:13071317, 1996.

STARK, J.M. \& HART, S.C. High rates of nitrification and nitrate turnover in undisturbed coniferous forests. Nature, 385:61-64, 1997.

TEDESCO, M.J.; VOLKWEISS, S.J. \& BOHMEN, H. Análises de solo, plantas e outros materiais. 2.ed., Porto Alegre, Universidade Federal do Rio Grande do Sul, 1995. 174p. (Boletim Técnico de Solos, 5)

WALSH, L.M. \& MURDOCK, J.T. Native fixed ammonium and fixation of applied ammonium in several Wisconsin soils. Soil Sci., 89:183-192, 1960.

WATSON, C.J. \& POLAND, P. Change in the balance of ammonium-N and nitrate- $\mathrm{N}$ content in soil under grazed grass swards over 7 years. Grass. Forage Sci., 54:248254, 1999.

WOLDENDORP, J.W. \& LAANBROEK, H.J. Activity of nitrifiers in relation to nitrogen nutrition of plants in natural ecosystems. Plant Soil, 115:217-228, 1989.

WONG, M.T.F. \& NORTCLIFF, S. Seasonal fluctuations of native available $\mathrm{N}$ and soil management implications. Fert. Res., 42:13-26, 1995. 